\title{
An Algebraic Method on the Eigenvalues and Stability of Delayed Reaction-Diffusion Systems
}

\author{
Jian $\mathrm{Ma}^{1,2}$ and Baodong Zheng ${ }^{2}$ \\ ${ }^{1}$ Department of Mathematics, Northeast Forestry University, Harbin 150040, China \\ ${ }^{2}$ Department of Mathematics, Harbin Institute of Technology, Harbin 150001, China
}

Correspondence should be addressed to Baodong Zheng; zbd@hit.edu.cn

Received 2 April 2013; Accepted 8 July 2013

Academic Editor: Xiang Ping Yan

Copyright (c) $2013 \mathrm{~J}$. Ma and B. Zheng. This is an open access article distributed under the Creative Commons Attribution License, which permits unrestricted use, distribution, and reproduction in any medium, provided the original work is properly cited.

\begin{abstract}
The eigenvalues and stability of the delayed reaction-diffusion systems are considered using the algebraic methods. Firstly, new algebraic criteria to determine the pure imaginary eigenvalues are derived by applying the matrix pencil and the linear operator methods. Secondly, a practical checkable criteria for the asymptotic stability are introduced.
\end{abstract}

\section{Introduction}

The reaction-diffusion system is a semilinear partial differential equation, which has been used for the study of morphogenesis, population dynamics, and autocatalytic oxidation reactions. The early reaction-diffusion models were constructed to describe the process of chemical reaction. For example, the Brusselator reaction-diffusion system and its improved systems had been researched by many scholars $[1,2]$. Besides, during the past years, many results about the stability, steady state bifurcation, and the Hopf bifurcation on the reaction-diffusion systems had been derived [3-8]. From those results, we see that most of them had been mainly focused on two methods: the analytical methods and the numerical methods. The analytical methods, such as the center manifold theorem, the normal form theory, and the Laplace transformation, were mainly used to research the analytic solutions and dynamical property. The numerical methods, such as the Runge-Kutta methods and linear multistep methods, paid close attention to checking the changes and convergence of solutions. For example, Wu determined the direction and stability of periodic solutions occurring through the Hopf bifurcation by the center manifold theory and the normal form theory, which are the classic analytical methods in functional differential systems [1]. Wei et al. in papers [3-5] demonstrated the bifurcation and stability of different reaction-diffusion systems.
In this paper, we will introduce a new algebraic method. In fact, in the past recent years, many algebraic methods were applied to the functional differential systems, such as matrix theory, polynomial theory, the Lie group, and algebraic system. In particular for the study on the more complex systems, such as the high dimensional functional differential systems, the algebraic methods have their advancement. So in the following, we will discuss the delayed reaction-diffusion systems by the algebraic methods. We will study a general delayed reaction-diffusion system with spatial domain $\Omega$ in the following form:

$$
\begin{aligned}
\frac{\partial u_{1}}{\partial t} & =d_{1} \Delta u_{1}+f_{1}\left(u_{i} ; u_{i}(x, t-\tau)\right) \\
\frac{\partial u_{2}}{\partial t} & =d_{2} \Delta u_{2}+f_{2}\left(u_{i} ; u_{i}(x, t-\tau)\right) \\
& \vdots \\
\frac{\partial u_{m}}{\partial t} & =d_{m} \Delta u_{m}+f_{m}\left(u_{i} ; u_{i}(x, t-\tau)\right) \\
& x \in \Omega, \quad t>0, \quad i=1,2, \ldots, m,
\end{aligned}
$$

where the spatial domain $\Omega$ is restricted to one-dimensional interval $(0, \pi)$, that is, $\Omega=(0, \pi)$. Also $u_{i}=u_{i}(x, t)>0(i=$ $1,2, \ldots, m)$ stands for the concentrations of $m$ reactants or 
densities of $m$ species. $\Delta$ denotes the Laplace operator and $\Delta u_{i}=\partial^{2} u_{i} / \partial x^{2} . d_{i}(i=1,2, \ldots, m)$ denotes the diffusion coefficients of $u_{i}$, respectively. This paper will consider (1) subject to the origin condition and the homogeneous Neumann boundary condition on spatial domain $\Omega$ :

$$
\begin{aligned}
& u_{i}(x, 0)=u_{i}(x), \quad x \in \Omega \\
& \frac{\partial u_{i}(x, t)}{\partial x}=0, \quad x=0, \pi, t>0 \\
& i=1,2, \ldots, m .
\end{aligned}
$$

For the system (1) and the determined condition (2), we suppose that $f_{i}(0)=0$, for any $t \geq 0$, and $f_{i}$ is $C^{k}, k \geq 5$. That is to say, the origin point $(0,0, \ldots, 0)$ is an equilibrium of system (1) and (2). So the matrix form of system (1) can be written as

$$
\frac{\partial U(x, t)}{\partial t}=\widetilde{\Delta} U(x, t)+F(U(x, t), U(x, t-\tau)),
$$

where

$$
\begin{aligned}
& U(x, t)=\left(\begin{array}{c}
u_{1}(x, t) \\
u_{2}(x, t) \\
\vdots \\
u_{m}(x, t)
\end{array}\right), \\
& \widetilde{\Delta}=\left(\begin{array}{cccc}
d_{1} \Delta & 0 & \cdots & 0 \\
0 & d_{2} \Delta & \cdots & 0 \\
\vdots & \vdots & \ddots & \vdots \\
0 & 0 & \cdots & d_{m} \Delta
\end{array}\right), \quad F=\left(\begin{array}{c}
f_{1} \\
f_{2} \\
\vdots \\
f_{m}
\end{array}\right) .
\end{aligned}
$$

The linearization of system (3) at origin point $(0,0, \ldots, 0)$ is

$$
\frac{\partial U(x, t)}{\partial t}=\widetilde{\Delta} U(x, t)+A U(x, t)+B U(x, t-\tau) .
$$

Furthermore, it is well known that the eigenvalue problem

$$
\psi^{\prime \prime}+\lambda \psi=0, \quad x \in(0, \pi), \psi^{\prime}(0)=\psi^{\prime}(\pi)=0
$$

has eigenvalues $\lambda_{n}=n^{2},(n=0,1,2, \ldots)$, with corresponding eigenfunctions $\psi_{n}=\cos n x$. Then eigenvalues of $\widetilde{\Delta}$ are $\left\{-d_{1} n^{2},-d_{2} n^{2}, \ldots,-d_{m} n^{2}\right\}, n=0,1,2, \ldots$ Let

$$
Y=\sum_{n=0}^{\infty} \cos n x \cdot y, \quad y=\left(\begin{array}{c}
y_{1 n} \\
y_{2 n} \\
\vdots \\
y_{m n}
\end{array}\right)
$$

Then $Y$ is an eigenfunction for (5) with eigenvalue $s$. By straightforward analysis, we obtain

$$
p_{n}(s, \tau) y=\left[s E-D_{n}-A-B e^{-s \tau}\right] y=0, \quad n=0,1,2, \ldots,
$$

where $D_{n}=\operatorname{diag}\left(-d_{1} n^{2},-d_{2} n^{2}, \ldots,-d_{m} n^{2}\right)$. So we can find all of the eigenvalues of system (5) from (8). Certainly, the stability, and Hopf bifurcation of system (1) can be described by studying the linear system (5).

The remaining parts of the paper are structured in the following way. In Section 2, we demonstrated the critical condition on the delay $\tau$ of the system (1) and got the algebraic criteria for determining the pure imaginary eigenvalues. In Section 3, we researched the stability and the Hopf bifurcation of the delayed reaction-diffusion equation (39) with Neumann boundary condition and derived the corresponding algebraic criteria. At last, we described a specific reaction-diffusion equation and simulated the results by MATLAB.

\section{Algebraic Criteria for Determining the Pure Imaginary Eigenvalues}

Firstly, we research an ordinary differential equation

$$
\begin{aligned}
& \dot{X}(t)=A_{k} X(t)+B Y(t) \\
& \dot{Y}(t)=-X(t) B^{\mathrm{T}}-Y(t) A_{k}^{\mathrm{T}},
\end{aligned}
$$

where $A_{k}, B \in \mathbb{R}^{n \times n}, X, Y \in \mathbb{C}^{n \times n}$. Let $V$ denote the vector space $V=\mathbb{C}^{n \times n} \times \mathbb{C}^{n \times n}$, let $I$ denote the identity operator on $V$, and let operator $\mathbf{M}$ on $V$ be given by

$$
\mathbf{M}\left(\begin{array}{c}
X \\
Y
\end{array}\right)=\left(\begin{array}{c}
A_{k} X+B Y \\
-X B^{\mathrm{T}}-Y A_{k}^{\mathrm{T}}
\end{array}\right), \quad \forall X, Y \in \mathbb{C}^{n \times n}
$$

With $Z(t)=\left(\begin{array}{c}X(t) \\ Y(t)\end{array}\right)$, the system (9) can be written as

$$
\dot{Z}(t)=\mathbf{M} Z(t) \text {. }
$$

Suppose that $\widetilde{Z}(t)=\left(\begin{array}{c}\widetilde{X} \\ \widetilde{Y}\end{array}\right)=\left(\begin{array}{c}X_{0} e^{s t} \\ Y_{0} e^{s t}\end{array}\right)$ is a matrix solution of the system (9). We have

$$
\begin{aligned}
& \left(s E-A_{k}\right) \widetilde{X}-B \tilde{Y}=0 \\
& \widetilde{X} B^{\mathrm{T}}+\widetilde{Y}\left(s E+A_{k}^{\mathrm{T}}\right)=0 .
\end{aligned}
$$

For any complex $s$, let $\mathbf{T}=\mathbf{T}(s)$ be the operator $\mathbf{T}=s \mathbf{I}-\mathbf{M}$. Then

$$
\mathbf{T}\left(\begin{array}{l}
X \\
Y
\end{array}\right)=\left(\begin{array}{c}
\left(s E-A_{k}\right) X-B Y \\
X B^{\mathrm{T}}+Y\left(s E+A_{k}^{\mathrm{T}}\right)
\end{array}\right), \quad \forall X, Y \in \mathbb{C}^{n \times n}
$$

For any complex $s$, let $\mathbf{T}^{+}=\mathbf{T}^{+}(s): V \rightarrow V$, satisfying

$$
\mathbf{T}^{+}\left(\begin{array}{l}
X \\
Y
\end{array}\right)=\left(\begin{array}{c}
X\left(s E+A_{k}^{\mathrm{T}}\right)+B Y \\
-X B^{\mathrm{T}}+\left(s E-A_{k}\right) Y
\end{array}\right), \quad \forall X, Y \in \mathbb{C}^{n \times n}
$$

For any complex $s$, let $\Lambda=\Lambda(s)$, satisfying

$$
\Lambda X=\left(s E-A_{k}\right) X\left(s E+A_{k}^{\mathrm{T}}\right)+B X B^{\mathrm{T}}, \quad \forall X \in \mathbb{C}^{n \times n} .
$$

By simple computations, we can get

$$
\begin{aligned}
& \left(s E-A_{k}\right) X\left(s E+A_{k}^{\mathrm{T}}\right)+B X B^{\mathrm{T}}=X_{0}\left(s E+A_{k}^{\mathrm{T}}\right)+B Y_{0} \\
& \left(s E-A_{k}\right) Y\left(s E+A_{k}^{\mathrm{T}}\right)+B Y B^{\mathrm{T}}=-X_{0} B^{\mathrm{T}}+\left(s E-A_{k}\right) Y_{0} .
\end{aligned}
$$


By the operator language, that is,

$$
\mathbf{T}^{+} \mathbf{T}\left(\begin{array}{l}
X \\
Y
\end{array}\right)=\left(\begin{array}{l}
\Lambda X \\
\Lambda Y
\end{array}\right)
$$

In the following, we will convert matrix ordinary differential equation (10) to vector form. Let $\xi$ be the elementary transform, $\xi: \mathbb{C}^{n \times n} \rightarrow \mathbb{C}^{n^{2}}$. That is,

$$
\xi A=\left(\begin{array}{c}
a_{1}^{\mathrm{T}} \\
\vdots \\
a_{n}^{\mathrm{T}}
\end{array}\right), \quad \forall A=\left(\begin{array}{c}
a_{1} \\
\vdots \\
a_{n}
\end{array}\right), a_{i}^{\mathrm{T}} \in \mathbb{C}^{n}, i=1,2, \ldots, n .
$$

Let $x=\xi X, y=\xi Y, z=\left(\begin{array}{l}x \\ y\end{array}\right)$, and

$$
I_{0}=\left(\begin{array}{cc}
E \otimes E & 0 \\
0 & E \otimes E
\end{array}\right), \quad M_{0}=\left(\begin{array}{cc}
A_{k} \otimes E & B \otimes E \\
-E \otimes B & -E \otimes A_{k}
\end{array}\right) .
$$

Using the property of the Kronecker product, we have

$$
\xi(A X B)=A \otimes B^{\mathrm{T}} \xi X,
$$

where $A, B, X \in \mathbb{C}^{n \times n}$. So (11) can be written as

$$
I_{0} \dot{z}(t)=M_{0} z(t) \text {. }
$$

Similarly, by denoting $T_{0}=T_{0}(s), T_{0}^{+}=T_{0}^{+}(s)$, and $\Lambda_{0}=$ $\Lambda_{0}(s)$ as follows:

$$
\begin{aligned}
& T_{0}=T_{0}(s)=\left(\begin{array}{cc}
\left(s E-A_{k}\right) \otimes E & -B \otimes E \\
E \otimes B & E \otimes\left(s E+A_{k}\right)
\end{array}\right) \\
&=s I_{0}-M_{0}, \\
& T_{0}^{+}=T_{0}^{+}(s)=\left(\begin{array}{cc}
E \otimes\left(s E+A_{k}\right) & B \otimes E \\
-E \otimes B & \left(s E-A_{k}\right) \otimes E
\end{array}\right), \\
& \Lambda_{0}=\Lambda_{0}(s)=\left(s E-A_{k}\right) \otimes\left(s E+A_{k}\right)+B \otimes B,
\end{aligned}
$$

we have

$$
T_{0}^{+} T_{0}\left(\begin{array}{l}
x \\
y
\end{array}\right)=\left(\begin{array}{l}
\Lambda_{0} x \\
\Lambda_{0} y
\end{array}\right), \quad \text { that is, } T_{0}^{+} T_{0}=\left(\begin{array}{cc}
\Lambda_{0} & 0 \\
0 & \Lambda_{0}
\end{array}\right) .
$$

Lemma 1. For all complex $s$, $\operatorname{det} T_{0}^{+}(s)=\operatorname{det} T_{0}(s)$, and $s o$ $\operatorname{det} T_{0}(s)= \pm \operatorname{det} \Lambda_{0}(s)$.

Proof. Let $T_{0}=\left(\begin{array}{ll}a & b \\ c & d\end{array}\right)$, where $a=\left(s E-A_{k}\right) \otimes E, b=-B \otimes E$, $c=E \otimes B$, and $d=E \otimes\left(s E+A_{k}\right)$. Noting the Kronecker product identities $(I \otimes M)(N \otimes I)=(N \otimes I)(I \otimes M)=N \otimes M$, we have $d b=b d, b c=c b$. By regularity of $\left(E, A_{k}\right)$, we know that $s E+A_{k}$ is nonsingular for enough complex $s$. So by the property of the polynomial and easy computations, we can get that $\operatorname{det} T_{0}^{+}(s)=\operatorname{det} T_{0}(s)$ for any complex $s$. Elsewhere,

$$
T_{0}^{+} T_{0}=\left(\begin{array}{cc}
\Lambda_{0} & 0 \\
0 & \Lambda_{0}
\end{array}\right)
$$

so

$$
\left(\operatorname{det} T_{0}\right)^{2}=\operatorname{det} T_{0}^{+} \operatorname{det} T_{0}=\left(\operatorname{det} \Lambda_{0}\right)^{2}
$$

Thus

$$
\operatorname{det} T_{0}(s)= \pm \operatorname{det} \Lambda_{0}(s)
$$

Theorem 2. Any pure imaginary eigenvalue of the system (5) or the system (1) is a zero point of $\operatorname{det} \Lambda_{0}(s)$ and thus also one of the eigenvalues of the matrix pencil $\left(I_{0}, M_{0}\right)$.

Proof. For (8), let $D_{k}+A=A_{k}, k=0,1,2, \ldots$ Then we have

$$
p_{k}\left(s, e^{-s \tau}\right)=s E-A_{k}-B e^{-s \tau} \text {. }
$$

Let $s=i w$ be a pure imaginary eigenvalue of the system (5), let $y$ be associated eigenvector, and let $\|y\|=1$. We have $p_{k}\left(s, e^{-s \tau}\right) y=0$. By conjugating and transforming, we can get

$$
\left(s E-A_{k}\right) y y^{*}\left(s E+A_{k}^{\mathrm{T}}\right)+B y y^{*} B^{\mathrm{T}}=0 .
$$

Via the elementary transform $\xi$, we get

$$
\left[\left(s E-A_{k}\right) \otimes\left(s E+A_{k}\right)+B \otimes B\right] \xi\left(y y^{*}\right)=0 .
$$

That is $\Lambda_{0}(s) u=0, u=\xi\left(y y^{*}\right)$. We know that $\operatorname{det} \Lambda_{0}(s)=0$, and so,

$$
\operatorname{det}\left(s I_{0}-M_{0}\right)= \pm \operatorname{det} \Lambda_{0}(s)=0 .
$$

From Theorem 2, we know that all of the pure imaginary eigenvalues of the system (1) are zero points of the algebraic equation

$$
\operatorname{det}\left[\left(s E-A_{k}\right) \otimes\left(s E+A_{k}\right)+B \otimes B\right]=0 .
$$

So the pure imaginary eigenvalues of the system (1) or (5) can be computed via the algebraic equation (31). In fact, (31) is usually called a polynomial eigenvalue problem. The classical and most widely used approach to research the polynomial eigenvalue problems is linearization, where the polynomial is converted into a larger matrix pencil with the same eigenvalues. There are many forms for the linearization, but the companion form is most typically commission [9]. So we have the following results.

Theorem 3. The pure imaginary eigenvalues of the system (5) are the general eigenvalues of matrix pencil $(P, Q)$, where

$$
P=\left(\begin{array}{ll}
I & 0 \\
0 & I
\end{array}\right), \quad Q=\left(\begin{array}{cc}
-G & -H \\
I & 0
\end{array}\right)
$$

$I=E \otimes E, G=\left(-A_{k}\right) \oplus A_{k}$, and $H=B \otimes B$. 
Proof. Equation (31) can be rewritten as

$$
\left[E \otimes E s^{2}+\left[E \otimes A_{k}-A_{k} \otimes E\right] s+B \otimes B\right] v=0
$$

where $v=\xi\left(y y^{*}\right)$. For $-A_{k} \otimes E+E \otimes A_{k}=\left(-A_{k}\right) \oplus A_{k}$, then

$$
\left[E \otimes E s^{2}+\left[\left(-A_{k}\right) \oplus A_{k}\right] s+B \otimes B\right] v=0 .
$$

Let $I=E \otimes E, G=\left(-A_{k}\right) \oplus A_{k}$, and $H=B \otimes B$; then we have

$$
\left[I s^{2}+G s+H\right] v=0 .
$$

Equation (35) is a quadratic eigenvalue problem, and its linearization is

$$
\left[s\left(\begin{array}{cc}
I & 0 \\
0 & I
\end{array}\right)-\left(\begin{array}{cc}
-G & -H \\
I & 0
\end{array}\right)\right] u=0,
$$

where $u=\left(\begin{array}{c}s v \\ v\end{array}\right)$. So let

$$
P=\left(\begin{array}{ll}
I & 0 \\
0 & I
\end{array}\right), \quad Q=\left(\begin{array}{cc}
-G & -H \\
I & 0
\end{array}\right) .
$$

Then $(s P-Q) u=0$ has nonzero solutions if and only if

$$
\operatorname{det}(s P-Q)=0 \text {. }
$$

So $s$ is the general eigenvalue of matrix pencil $(P, Q)$.

Suppose that the system (1) is stable at the initial time. Then the stability of the system will change as the positive real part root of characteristic equation emerged for the different parameter values of system (1). So the first pure imaginary eigenvalue is the critical condition and plays an important role in researching the stability and the Hopf bifurcation of the delayed reaction-diffusion equation. From the previous results, we can get all of the pure imaginary eigenvalues for a delayed reaction-diffusion equation. Here let the time delay $\tau$ be the parameter. Then we can get the stable or unstable condition of the delayed reaction-diffusion equation for different parameter values.

\section{The Stability and the Hopf Bifurcation of a Delayed Reaction-Diffusion Equation}

In the recent years, scholars discussed many different reaction-diffusion systems [10-12]. For different systems, they expounded different results to justify the stability. Here we will study a general high-dimensional reaction-diffusion equation with single delay. The considered reaction-diffusion system is

$$
\begin{array}{ll}
\frac{\partial u_{1}}{\partial t}=d_{1} \Delta u_{1}+f_{1}\left(u_{i}(x, t) ; u_{i}(x, t-\tau)\right) & x \in \Omega, t>0 \\
\frac{\partial u_{2}}{\partial t}=d_{2} \Delta u_{2}+f_{2}\left(u_{i}(x, t) ; u_{i}(x, t-\tau)\right) & x \in \Omega, t>0 \\
\vdots & \\
\frac{\partial u_{m}}{\partial t}=d_{m} \Delta u_{m}+f_{m}\left(u_{i}(x, t) ; u_{i}(x, t-\tau)\right) & x \in \Omega, t>0 \\
\frac{\partial u_{i}(x, t)}{\partial n}=0 \quad x \in \partial \Omega, t>0 & \\
u_{i}(x, t)=\varphi_{i}(x, t) \quad x \in \Omega, t \in[-\tau, 0], &
\end{array}
$$

where $i=1,2, \ldots, m$ and $\Omega=[0, \pi]$ is a bounded connected domain in $R$ with smooth boundary $\partial \Omega$. The functions $f_{i}$ are continuously differentiable, and $\partial u_{i} / \partial n$ is the out normal derivative of $u_{i}$. We assume that $u_{i}^{*}>0$ such that

$$
f_{i}\left(u_{1}^{*}, u_{2}^{*}, \ldots, u_{m}^{*}\right)=0 .
$$

That is to say, $\left(u_{1}^{*}, u_{2}^{*}, \ldots, u_{m}^{*}\right)$ is the positive equilibrium of system (39). The linearization of system (39) is

$$
\frac{\partial X(x, t)}{\partial t}=\tilde{\Delta} X(x, t)+A X(x, t)+B X(x, t-\tau),
$$

$$
X(x, t)=\left(\begin{array}{c}
u_{1}(x, t) \\
u_{2}(x, t) \\
\vdots \\
u_{m}(x, t)
\end{array}\right) \text {, }
$$

$$
\widetilde{\Delta}=\left(\begin{array}{cccc}
d_{1} \Delta & 0 & \cdots & 0 \\
0 & d_{2} \Delta & \cdots & 0 \\
\vdots & \vdots & \ddots & \vdots \\
0 & 0 & \cdots & d_{m} \Delta
\end{array}\right),
$$$$
A=\left(\begin{array}{cccc}
\frac{\partial f_{1}}{\partial u_{1}} & \frac{\partial f_{1}}{\partial u_{2}} & \cdots & \frac{\partial f_{1}}{\partial u_{m}} \\
\frac{\partial f_{2}}{\partial u_{1}} & \frac{\partial f_{2}}{\partial u_{2}} & \cdots & \frac{\partial f_{2}}{\partial u_{m}} \\
\vdots & \vdots & \ddots & \vdots \\
\frac{\partial f_{m}}{\partial u_{1}} & \frac{\partial f_{m}}{\partial u_{2}} & \cdots & \frac{\partial f_{m}}{\partial u_{m}}
\end{array}\right)_{\left(u_{1}^{*}, u_{2}^{*}, \ldots, u_{m}^{*}\right)},
$$$$
B=\left(\begin{array}{cccc}
\frac{\partial f_{1}}{\partial u_{1 \tau}} & \frac{\partial f_{1}}{\partial u_{2 \tau}} & \cdots & \frac{\partial f_{1}}{\partial u_{m \tau}} \\
\frac{\partial f_{2}}{\partial u_{1 \tau}} & \frac{\partial f_{2}}{\partial u_{2 \tau}} & \cdots & \frac{\partial f_{2}}{\partial u_{m \tau}} \\
\vdots & \vdots & \ddots & \vdots \\
\frac{\partial f_{m}}{\partial u_{1 \tau}} & \frac{\partial f_{m}}{\partial u_{2 \tau}} & \cdots & \frac{\partial f_{m}}{\partial u_{m \tau}}
\end{array}\right)_{\left(u_{1}^{*}, u_{2}^{*}, \ldots, u_{m}^{*}\right)}
$$ 
where $u_{i}=u_{i}(x, t)$ and $u_{i \tau}=u_{i}(x, t-\tau)$. For simplicity we suppose that the eigenvalues of the Laplace operator $\Delta$ by the Neumann boundary condition are simple. From Section 1, we know that the eigenvalues of $\Delta$ in $(0, \pi)$ with the Neumann boundary condition are $\mu_{n}=-n^{2}, n=0,1,2, \ldots$. Let $s$ denote an eigenvalue of system (39). Then there exists a nonzero vector $y \in \operatorname{Dom}(\widetilde{\Delta})$ such that

$$
p(s, \tau, n) y=\left(s E-D_{n}-A-B e^{-s \tau}\right) y=0 .
$$

The stability of the system (39) is determined by

$$
\operatorname{det} p(s, \tau, n)=0 .
$$

We know that the system (39) is asymptotically stable when all of the eigenvalues of (42) are in the left part of the imaginary axis. Apparently, for nondiffusive case, that is, $d_{1}=d_{2}=\cdots=$ $d_{m}=0$, the system (39) becomes a delay differential equation, and the stability has been studied by many scholars. Here we mainly discuss the diffusive system. Let

$$
S(\tau, n)=\{s \in C \mid \operatorname{det} p(s, \tau, n)=0\} .
$$

Firstly, when $\tau=0$, the system (39) becomes a partial differential equation with no delay. So we can get the stability in the following.

Lemma 4. For $\tau=0$, let $s$ be any element of the set $S(0, n)$. That is, $s \in \sigma\left(D_{n}+A+B\right)$.

(i) The system (39) is eigenmode $n$-independently stable if Res $<0$, for all $n \in N$;

(ii) else, the system (39) is eigen-moden-dependently stable if Res $<0$, for a fixed $n \in N$.

Next we research the delay-independent and the delaydependent stabilities of the system (39). Let $\sigma(P, Q)$ denote the set which contains all the general eigenvalues of matrix pencil $(P, Q)$. From (38) in Section 2, we have the following results.

Theorem 5. For any $\tau \geq 0$ and a fixed eigen-mode $n \in N$, if the coefficient matrices of the system (39) satisfy the following conditions:

(i) Res $<0, s \in \sigma\left(D_{n}+A+B\right)$,

(ii) Res $\neq 0, s \in \sigma(P, Q)$,

then the system (39) is asymptotically stable for all $\tau \geq 0$ with fixed eigen-mode n; for example, the stability is delayindependent and eigenmode n-dependent. On the other hand, if, for all eigen-mode $n$, the stability is delay independent, then stability of system (39) is delay independent and eigenmode nindependent.

Lemma 6. Given a fixed eigen-mode $n \in N$, let Res $<0$, $s \in \sigma\left(D_{n}+A+B\right)$. For all the pure imaginary roots $s=i w$ of (31) (or general pure imaginary eigenvalues of matrix pencil $(P, Q))$, one can get the critical value of delay parameter $\tau_{n}^{*}$ for the fixed eigen-mode $n$.
Conclusion. Suppose that the system (39) is eigen-mode $n$ independently stable at $\tau=0$, and for multiple eigen mode $n$, the critical values of delay parameter are $\tau_{n}^{*}, n=1,2, \ldots, k$. Let $\tau^{*}=\min \left\{\tau_{n}^{*} \mid n=1,2, \ldots, k\right\}$. One can get the minimal critical value of delay parameter $\tau^{*}$. When $\tau \in\left[0, \tau^{*}\right)$, the system (39) is asymptotically stable, and when $\tau \geq \tau^{*}$, the stability of system (39) changes. That is to say, the positive equilibrium of system (39) $\left(u^{*}, v^{*}\right)$ is delay dependently stable, and the system (39) first generates bifurcation at $\tau=$ $\tau^{*}$.

From the above theorems, we can discuss the stability and the Hopf bifurcation of system (39). So the dynamical character of a generic class of nonlinear reaction-diffusion system with the Neumann boundary condition can be studied by the algebraic methods. It is well known that reaction and diffusion of physical chemistry and chemical or biochemical species can produce all kinds of spatial patterns. Next we will give a general example.

Example 7. Consider a delayed reaction-diffusion equation with the Neumann boundary condition:

$$
\begin{aligned}
& \frac{\partial u}{\partial t}=d_{1} \Delta u+c-a u(x, t)+k \frac{u^{2}(x, t-\tau)}{v(x, t-\tau)} \quad x \in \Omega, t>0, \\
& \frac{\partial v}{\partial t}=d_{2} \Delta v+\gamma u^{2}(x, t-\tau)-b v(x, t) \quad x \in \Omega, t>0, \\
& \frac{\partial u(x, t)}{\partial x}=0, \quad \frac{\partial v(x, t)}{\partial x}=0, \quad x \in \partial \Omega, t>0, \\
& u(x, t)=u_{0}(x, t), \quad v(x, t)=v_{0}(x, t) \quad x \in \Omega, t \in[-\tau, 0],
\end{aligned}
$$

where $a, b, c, k$, and $\gamma$ especially denote the decay rate. This system is a biochemically mathematical model, such as the improved Gierer gene model. Apparently, $\left(u^{*}, v^{*}\right)=$ $\left((\gamma c+k b) / a \gamma,(\gamma c+k b)^{2} / a^{2} b \gamma\right)$ is the unique constant positive equilibrium of system (45). Here we will only consider the numerical case of $d_{1}=0.03, d_{2}=0.3, a=0.8, b=1$, $c=0.2, k=1$, and $\gamma=1$. Hence the positive equilibrium $\left(u^{*}, v^{*}\right)=(1.5,2.25)$. Then $(43)$ has no root in the right of the imaginary axis for $n \geq 2$, has two roots in the imaginary axis $s_{11} \doteq \pm 0.305 i$ and $s_{12} \doteq \pm 0.702 i$ for $n=1$, and has two roots in the imaginary axis $s_{01} \doteq \pm 0.648 i$ and $s_{02} \doteq \pm 0.803 i$ for $n=0$. The parameter $\tau$ has four sequences $\tau_{1} \doteq 1.625+2 k \pi / 0.305$, $\tau_{2} \doteq 6.898+2 k \pi / 0.702, \tau_{3} \doteq 0.424+2 k \pi / 0.648$, and $\tau_{4} \doteq 5.782+2 k \pi / 0.803, k=0,1, \ldots$ So the smallest Hopf bifurcation value is $\tau^{*}=\min \left\{\tau_{1}, \tau_{2}, \tau_{3}, \tau_{4}\right\}=0.424$. Then the unique positive equilibrium of system (45) is asymptotically stable when $\tau \in\left[0, \tau^{*}\right)$ and unstable when $\tau>\tau^{*}$. The simulation results are shown in Figures 1 and 2.

\section{Conclusion}

In this paper, we consider a general high-dimensional delayed reaction-diffusion system. By the algebraic methods, such as the matrix pencil and the linear operator, we discussed the eigenvalues and the stability of the delayed reaction-diffusion system (1) and (39). In fact, we only find the pure imaginary 


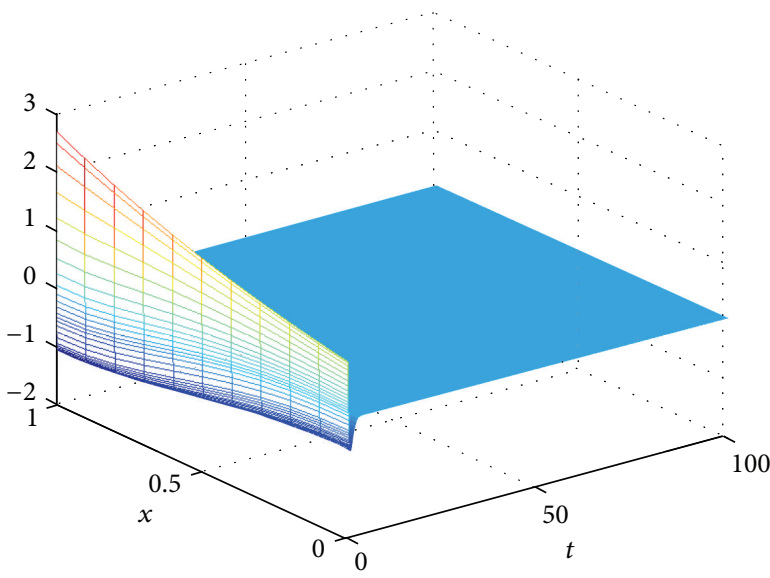

(a)

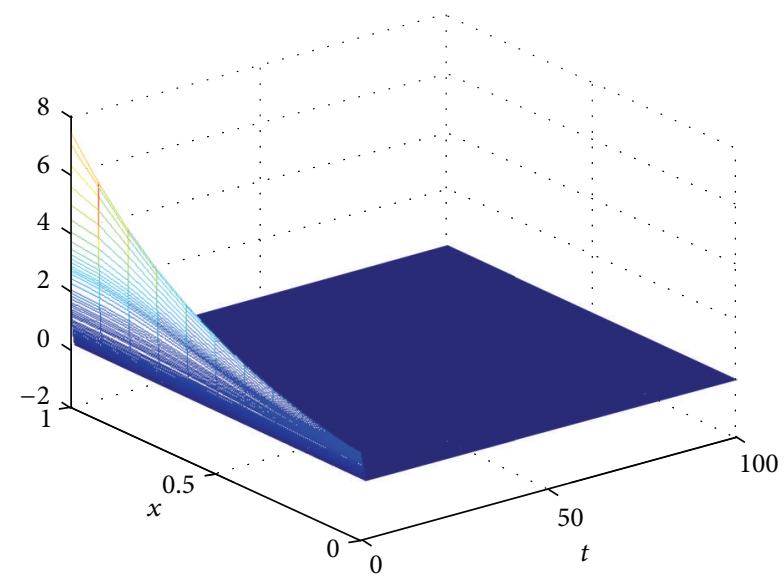

(b)

FIGURE 1: For system (45), when $\tau=0.2<\tau^{*}$, the equilibrium $\left(u^{*}, v^{*}\right)$ is locally asymptotically stable.

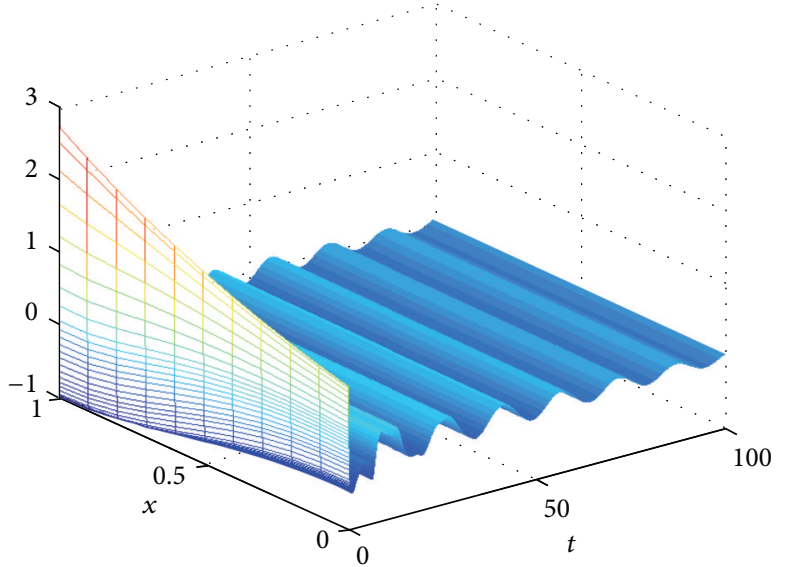

(a)

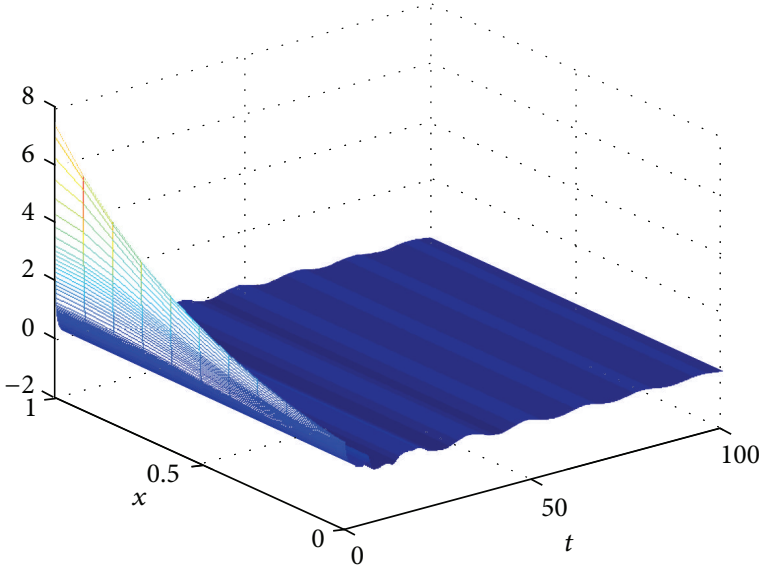

(b)

FIGURE 2: For system (45), when $\tau=6>\tau^{*}$, the equilibrium $\left(u^{*}, v^{*}\right)$ is unstable.

eigenvalues, which are a few parts of the infinite eigenvalues. So we can easily compute pure imaginary eigenvalues from the algebraic equation (31) by MATLAB. Certainly, applying the algebraic methods to analyze the dynamical properties of the reaction-diffusion system with delays is still a new and immature field, so we believe that the algebraic methods used to research the stability of the delayed reaction-diffusion systems would be of more interest in the future.

\section{Acknowledgment}

The research is funded by the Fundamental Research Funds for the Central Universities (DL12BB24).

\section{References}

[1] J. Wu, Theory and Applications of Partial Functional-Differential Equations, Springer, 1996.
[2] M. Ghergu, "Non-constant steady-state solutions for Brusselator type systems," Nonlinearity, vol. 21, no. 10, pp. 2331-2345, 2008.

[3] J. Fang, J. Wei, and X.-Q. Zhao, "Spatial dynamics of a nonlocal and time-delayed reaction-diffusion system," Journal of Differential Equations, vol. 245, no. 10, pp. 2749-2770, 2008.

[4] F. Yi, J. Wei, and J. Shi, "Diffusion-driven instability and bifurcation in the Lengyel-Epstein system," Nonlinear Analysis. Real World Applications, vol. 9, no. 3, pp. 1038-1051, 2008.

[5] F. Yi, J. Wei, and J. Shi, "Bifurcation and spatiotemporal patterns in a homogeneous diffusive predator-prey system," Journal of Differential Equations, vol. 246, no. 5, pp. 1944-1977, 2009.

[6] Y. You, "Global dynamics of the Brusselator equations," Dynamics of Partial Differential Equations, vol. 4, no. 2, pp. 167-196, 2007.

[7] C. C. Travis and G. F. Webb, "Existence, stability, and compactness in the $\alpha$-norm for partial functional differential equations," Transactions of the American Mathematical Society, vol. 240, pp. 129-143, 1978. 
[8] M. C. Memory, "Stable and unstable manifolds for partial functional differential equations," Transactions of the American Mathematical Society, vol. 200, pp. 395-418, 1974.

[9] D. S. Mackey, N. Mackey, C. Mehl, and V. Mehrmann, "Vector spaces of linearizations for matrix polynomials," SIAM Journal on Matrix Analysis and Applications, vol. 28, no. 4, pp. 971-1004, 2006.

[10] J. S. Dos Santos and M. A. Bená, "The delay effect on reactiondiffusion equations," Applicable Analysis, vol. 83, no. 8, pp. 807824, 2004.

[11] X.-P. Yan and W.-T. Li, "Stability of bifurcating periodic solutions in a delayed reaction-diffusion population model," Nonlinearity, vol. 23, no. 6, pp. 1413-1431, 2010.

[12] X. D. Lin, J. W.-H. So, and J. H. Wu, "Centre manifolds for partial differential equations with delays," Proceedings of the Royal Society of Edinburgh A, vol. 122, no. 3-4, pp. 237-254, 1992. 


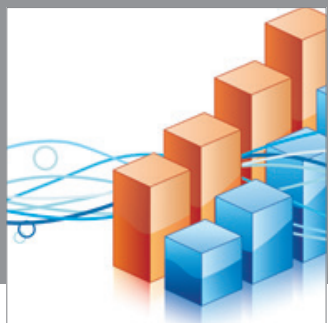

Advances in

Operations Research

mansans

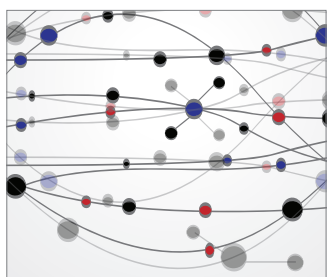

The Scientific World Journal
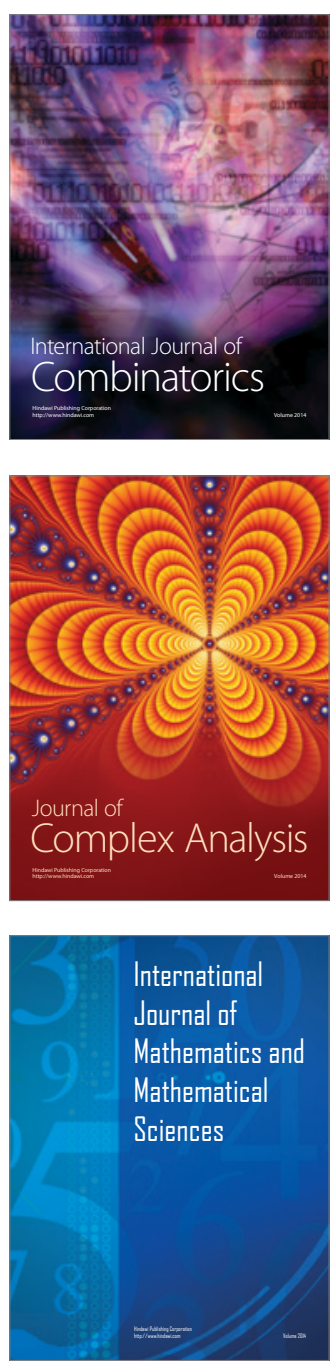
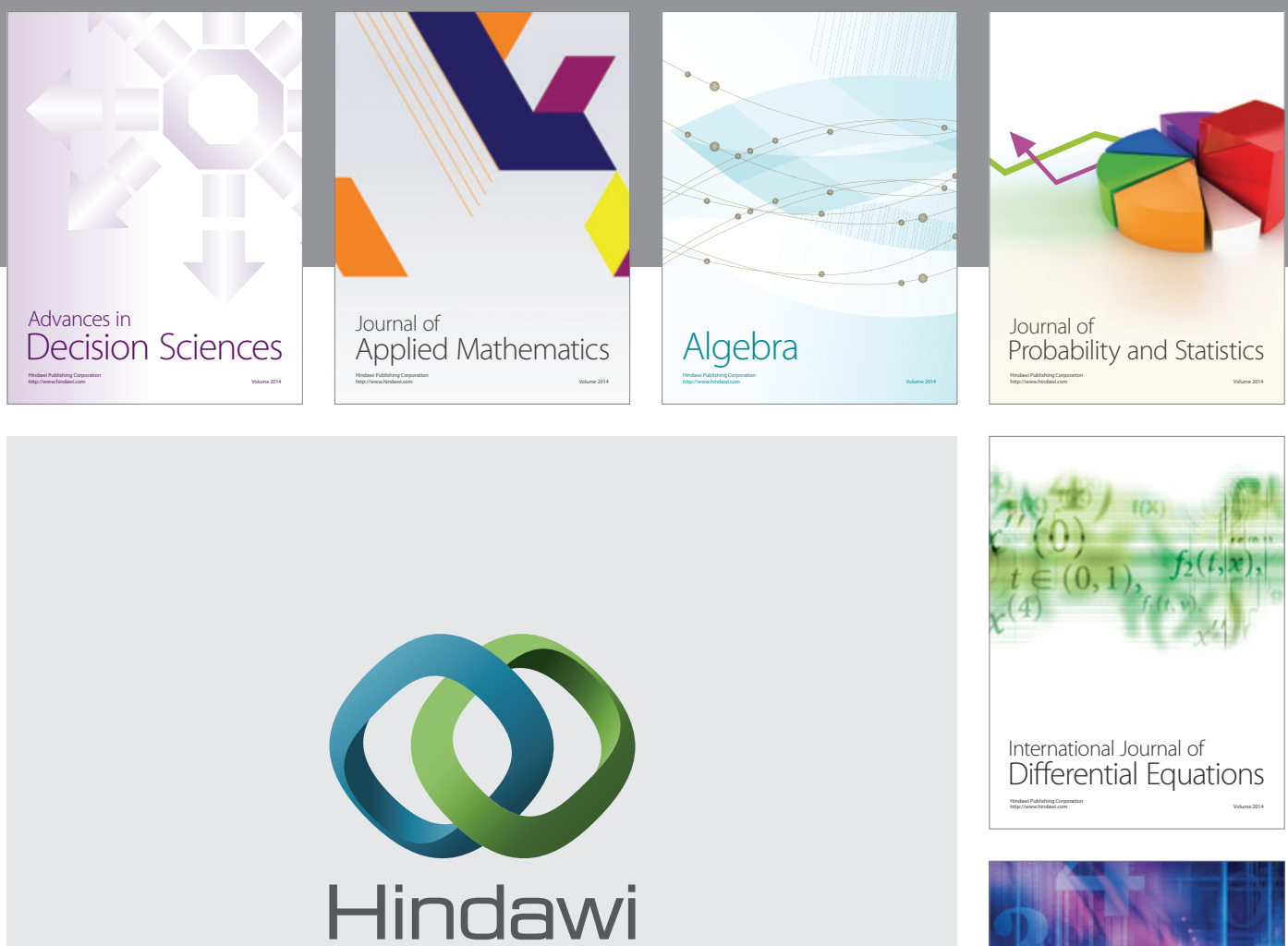

Submit your manuscripts at http://www.hindawi.com
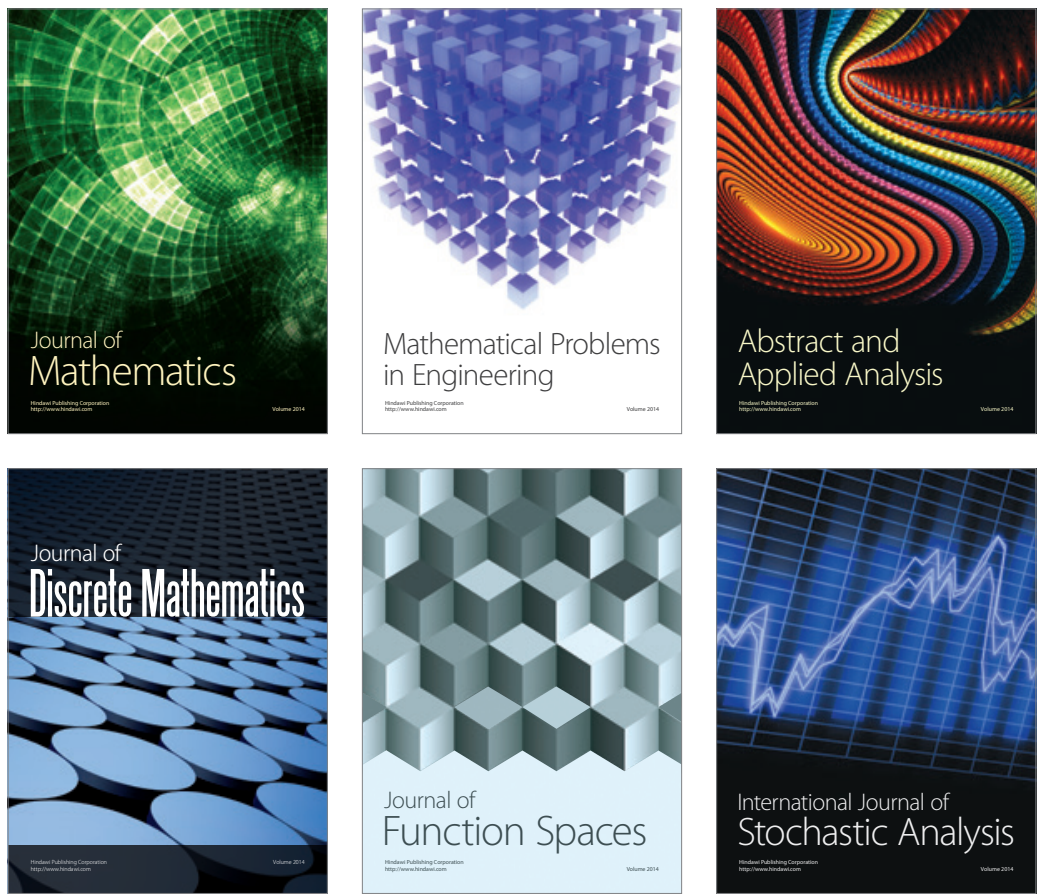

Journal of

Function Spaces

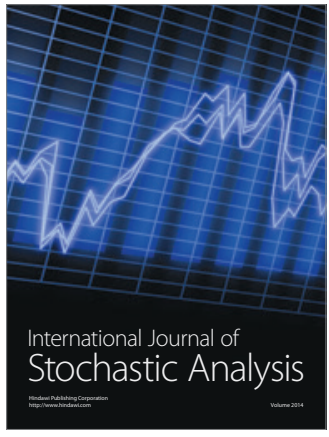

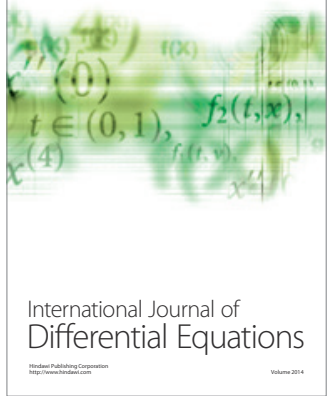
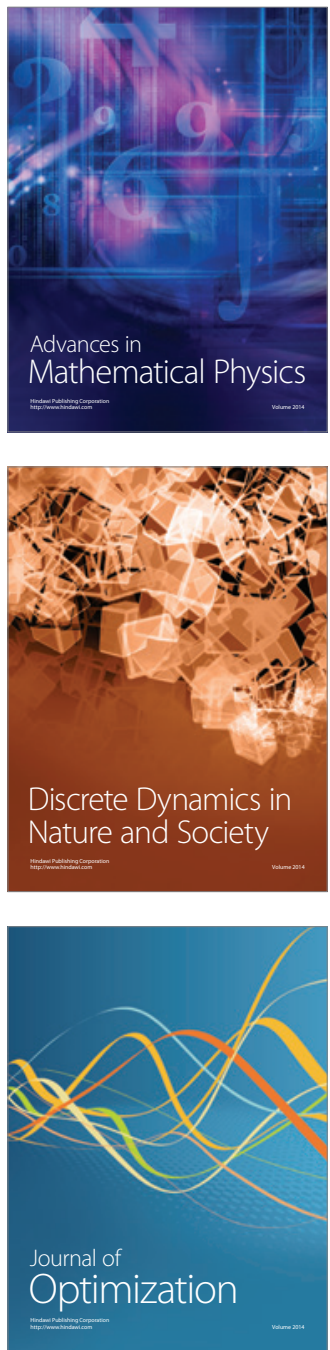